\title{
Castleman's Disease - A Rare Cause of Pyrexia of Unknown Origin (PUO): Case Report
}

\author{
Nusrat Sultana ${ }^{1}$,Rezaul Karim Chowdhury', Naseem Aktar Chowdhury ${ }^{3}$, Md Fariduddin $^{4}$, Javed-Jamal ${ }^{5}$,MAbul Hasanat ${ }^{4}$ \\ ${ }^{1}$ Medical Officer, ${ }^{2}$ MD Student, ${ }^{3}$ Professor, ${ }^{4}$ Associate Professor, Endocrine Wing, Department of Medicine, ${ }^{5}$ Department of Pathology \\ Bangabandhu Sheikh Mujib Medical University (BSMMU)
}

\begin{abstract}
:
Pyrexia of unknown origin (PUO) is a common problem in medical practice. Patients suffer from fever for long period but initial relevant investigations remain normal. Infection is the most common cause of PUO in developing countries. Other causes are lymphoma, myeloma, connective tissue diseases (CTD), sarcoidosis and malignancy etc. In our country, where no cause can be identified, a usual practice is to start anti-tubercular drugs. We report a patient who was suffering from prolonged fever and usual investigations were within normal limit except high erythrocyte sedimentation rate. In spite of having 6 months anti-tubercular drugs, the patient did not respond. Thorough examination revealed a paraaortic mass which was reconfirmed by ultrasound of abdomen. Laparotomy revealed a mesenteric lymph node that was removed. Histopathologically it was diagnosed as a case of CASTLEMAN'S DISEASE which is a rare cause of PUO. On removal of the lymph node, the patient improved dramatically.
\end{abstract}

Key words: Castleman's disease, PUO

[BSMMU J 2009; 2(2): 85-87]

\section{Introduction:}

Castleman's disease (CD) involves a massive nonmalignant proliferation of lymphoid tissue and typically presents as mediastinal mass. This uncommon condition was initially described among a small series of patients in 1956. ${ }^{1} \mathrm{CD}$ affects patients of varying ages and cases have been reported from adolescence into the seventh decade. ${ }^{2}$ Histologically CD are of hyaline vascular variant(80-90\%), plasma cell variant(10-20\%) and mixed histological appearance. Patients with hyaline vascular form of CD may exhibit no symptoms or only lymphadenopathy while the plasma cell variant typically present with fever, weight loss, rash, and anaemia. ${ }^{3}$

We report a 16-year-old girl who presented as pyrexia of unknown origin (PUO) i.e. with prolonged fever, single mesenteric lymphadenopathy and high erythrocyte sedimentation rate (ESR). Finally she was diagnosed as a case of localized CD. After removal of lymph node she became symptom free dramatically and ESR fell to normal level.

\section{Case report:}

A 16-year-old young girl presented with the complaints of high grade continued fever with anorexia, nausea, weight loss, night sweats for 18 months with no other

Address of Correspondence: Dr. M A Hasanat, Associate Professor of Endocrine Medicine, Department of Medicine, BSMMU, Dhaka1000, Bangladesh, E-mail: <hasanatdr@yahoo.com> systemic symptoms. With these complaints she was admitted in different hospitals and was treated as a case of enteric fever, malaria etc. She was also given a course of anti-tubercular therapy empirically for 6 months without any appreciable improvement. Finally she got herself admitted into Endocrine Wing of department of Medicine, BSMMU.

On admission her $\mathrm{Hb} \%$ was 8.5 gm/dl, ESR persistently high (over $100 \mathrm{~mm}$ fall in $1^{\text {st }}$ hour), total (TC) and differential (DC) blood counts were normal, peripheral blood film (PBF) showed microcytic hypochromic anemia with normal serum ferritin. There was no growth in blood culture, anti nuclear antibody was negative, chest $\mathrm{x}$-ray normal as well as renal and liver functions revealed no abnormality. Ultrasound of whole abdomen was indecisive. A thorough examination revealed a para-aortic palpable mass with ill defined margin, firm in consistency, not freely mobile but free from anterior abdominal wall. Other systemic examinations were unremarkable.

On repeat investigation after 2 weeks from admission her $\mathrm{Hb} \%$ was $9 \mathrm{gm} / \mathrm{dl}$, ESR-130 $\mathrm{mm}$ fall in $1^{\text {st }}$ hour, TC DC were normal, PBF- microcytic hypochromic anemia, C-reactive protin-116 mg/dl, chest X-ray- normal, urine for routine examination normal, ANA- negative, urine for katex testnormal, liver function tests, serum creatinine and random blood sugar were also normal, anti-HIV antibody was found negative. Ultrasound of whole abdomen revealed a single para-aortic lymph node $(5 \mathrm{~cm} \times 5 \mathrm{~cm})$. Neither blood culture nor urine culture showed any microbial growth. 
Finally laparotomy was done to explore the mesenteric lymph node (Fig-1). The lymph node was sent for histopathology which revealed large lymphoid follicles

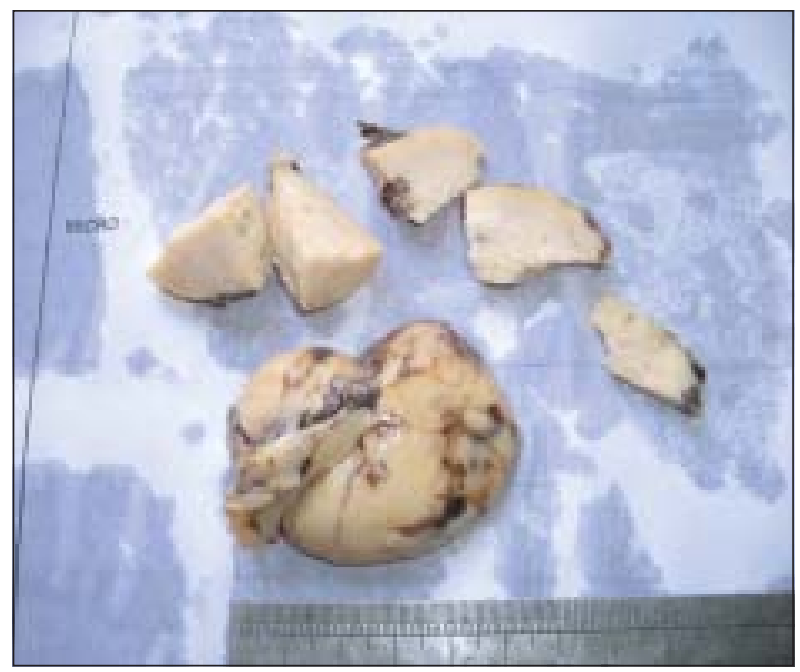

Fig-1: Single mesenteric lymph node.

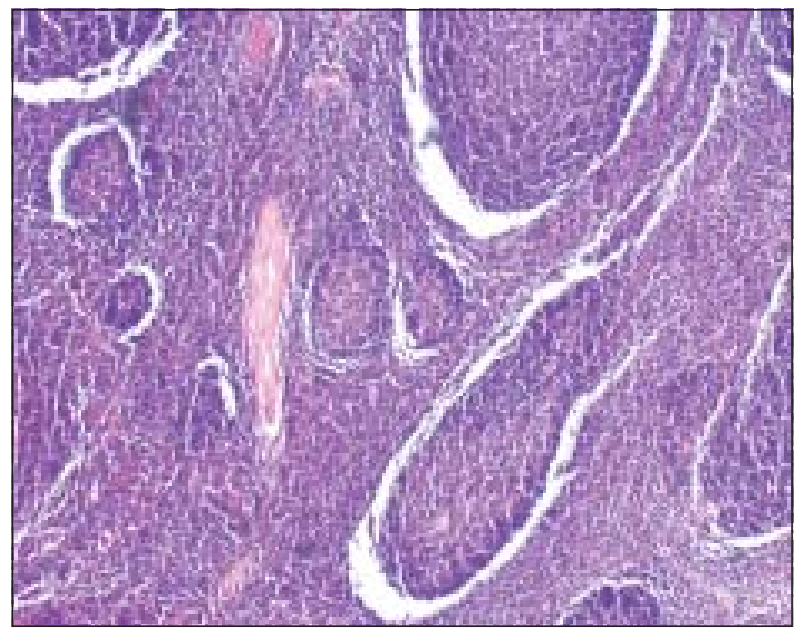

Fig-2: Lymph node of Castleman's disease displaying prominent central hyalinized blood vessel surrounded by concentric layer of follicular center cells.

with germinal centers. Few hyalinized blood vessels and many plasma cells were present both in germinal centers and interfollicular areas (fig-2). The follicles were surrounded by mantle zones mimicking the picture of plasma cell variant of Castleman's disease (fig-3).

We conclude that she was suffering from a localized Castleman's disease, plasma cell type. After removal of the lymph node her temperature subsided and all the constitutional symptoms gradually resolved, her ESR fell down to normal (ESR-15 mm in $1^{\text {st }}$ hour), $\mathrm{Hb} \%$ gradually

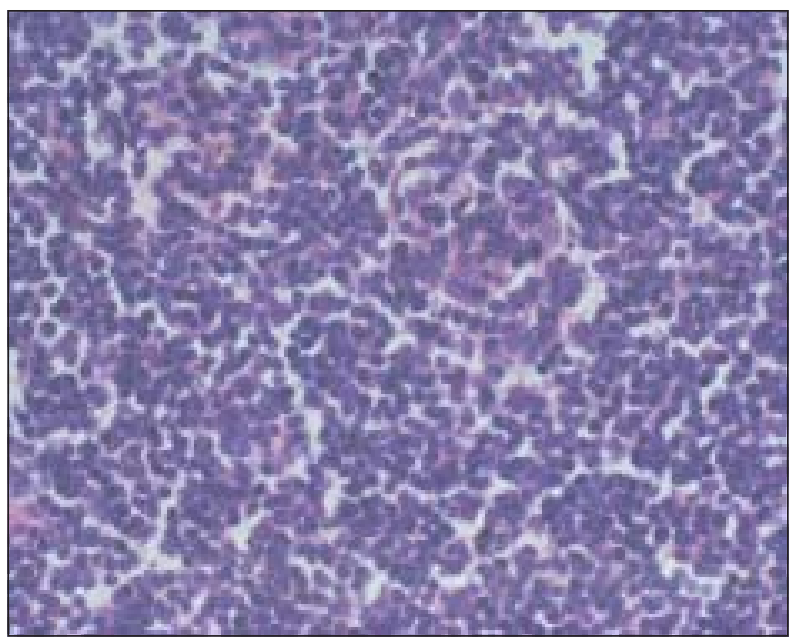

Fig-3: Lymph node of Castleman's disease depicting interfollicles with plasma cell proliferation.

increased to $11.0 \mathrm{gm} / \mathrm{dl}$, and she had remarkable clinical improvement.

\section{Discussion:}

Castleman's disease was first described by Dr Benjamin Castleman in 1956. The synonyms of this disease are giant lymph node hyperplasia and angiofollicular lymph node hyperplasia, a disease of lymph nodes and other related tissues. ${ }^{1}$ It is not a cancer but may give rise to a cancer at a later time. Treatments used for CD are the same as are used for people with other malignancies i.e. surgery, radiation therapy, and chemotherapy. The age of affected patients ranges between second to seventh decades. ${ }^{2}$

There are two main forms of CD-localized and multicentric. Localized or unicentric CD only involves a single lymph node and most often found in the chest and abdomen. Multicentric Castleman's disease affect more than one group of lymph nodes, and may also affect other organs containing lymphoid tissue. ${ }^{2}$ Pathological types of CD are- hyaline vascular variant, plasma cell variant and mixed type. The hyaline vascular variant exhibits prominent proliferation of small hyalinized follicles with marked interfollicular vascular proliferation, while the plasma cell variant exhibits hyperplasic germinal centers, sheets of plasma cells in the interfollicular region, proliferation of blood vessels and persistent sinuses. It is postulated that $10-20 \%$ of all cases are of the plasma cell variant, with a small percentage being of mixed histological appearance. The hyaline vascular variant tends to be localized and the plasma cell variant tends to be multicentric, but exceptions to this rule often occur. ${ }^{3} \mathrm{CD}$ affects children as well as adults. Young people are more likely to have the localized form. The multicentric form is more likely to affect older adults. ${ }^{2}$ 
The cause this disease is not yet discovered; however, a virus called human herpes virus (HHV-8) is associated with multicentric CD in many instances. ${ }^{4}$ Risk factors for $\mathrm{CD}$ are either genetic (inherited), life style related, or environmental. The only clear cut risk factor is HIV infection usually with multicentric type. ${ }^{5}$ An enlarged lymph node usually inside the chest or abdomen is the only problem for most people with the localized form of CD. Multicentric CD has more than one enlarged lymph node in the chest and abdomen, enlargement of liver, spleen or other organs also occur. People of either type of CD may have other symptoms and signs that include fever, anemia, weakness, night sweats, weight loss, loss of appetite, nausea and vomiting and nerve damage leading to numbness and weakness. These symptoms occur about twice as often in multicentric than in localized CD.

The diagnostic basis of CD is lymph node biopsy. Immunohistochemistry may be helpful in differentiating CD from lymphoma. Flow cytometry can help to determine whether lymph node swelling is caused by non Hodgkin's lymphoma, some other cancer or a non or precancerous disease like CD. Chest X-ray, CT scan, MRI, Gallium scan are important to classify the disease as localized or multicentric. $^{2}$

Localized CD is generally curable with surgical resection with or without radiotherapy. Symptoms such as fever and fatigue that are due to the CD go away after removal of the affected lymph node. Relapses are rare. Some patients with localized CD develop secondary amyloidosis, a condition in which abnormal proteins accumulate in the kidney, skin, and some other organs. This accumulation does not progress once the lymph node affected by CD is removed. $3,6-$ ${ }^{8}$ At present there is no consensus about the optimal management strategy of multicentric CD. Successful treatment of multicentric CD has been achieved using chemotherapy with or without prednisolone, given at the time of diagnosis. ${ }^{9}$ The chemotherapeutic agents used in the various case series yielding remission include doxorubicin, vincristine, cyclophosphamide, melphalan and chlorambucil. These have been used as single agent, together with steroid or in combination (CHOP). Azathioprine and bone marrow transplantation have also been attempted, especially following failure to $\mathrm{CHOP}$ but have yielded mixed result. ${ }^{3}$ Once localized, unicentric CD is surgically removed. The outcome is usually good and the disease may not grow back. ${ }^{3,6-8}$ However, the outcome with multicentric disease is not much satisfactory. Although treatment helps, cure is rare and the disease frequently recurs. ${ }^{9}$

\section{Contributors:}

Nusrat-Sultana and Rezaul Karim Chowdhury thoroughly examined and investigated the case under supervision of M Abul Hasanat and Professor Naseem Aktar Chowdhury. They prepared the initial manuscript and endorsed important and pertinent references. M Abul Hasanat and M Fariduddin finally formatted the case for publication. All of them are consultants of the Endocrine wing of department of Medicine of BSMMU. Javed Jamal made the histological diagnosis and helped with the photographs of slides. All the authors have gone through and contributed to the final manuscript.

\section{Acknowledgements:}

Authors acknowledge the kind help of Dr. Mohibul Aziz who was the surgical specialist and performed successful surgery that was resection of lymph node.

\section{References:}

1. Castleman B, Iverson I, Menendez VP. Localized mediastinal lymph-node hyperplasia resembling thymoma. Cancer. 1956; 9: 822-30.

2. Herrada J, Cabanillas F, Rice L, Manning J, Pugh W. Clinical behavior of localized and multicentric Castleman's disease. Annals of Internal Medicine. 1998; 128: 657-62.

3. Keller AR, Hochholzer L. Hyaline-vascular and plasma-cell types of giant lymph node hyperplasia of the mediastinum and other locations. Cancer. 1972; 29: p670.

4. Dupin N, Diss TL, Kellam P, Tulliez M, Du MQ, Sicard D, Weiss RA, Isaacson PG, Boshoff C. HHV-8 is associated with a plasmablastic variant of Castleman disease that is linked to HHV-8-positive plasmablastic lymphoma. Blood. 2000; 95: 1406-12.

5. Oksenhendler E, Duarte M, Soulier J, Cacoub P, Welker Y, Cadranel J. Multicentric Castleman's disease in HIV infection: a clinical and pathological study of 20 patients. AIDS. 1996; 10: 61-67.

6. Case records of the Massachusetts General Hospital; Weekly clinicopathological exercises. Case 39: A 66-year-old man with demyelinative neuropathy and a retroperitoneal mass. $N$ Engl J Med. 1990; 323: 895-908.

7. Lachant NA, Sun NC, Leong LA, Oseas RS, Prince HE. Multicentric angiofollicular lymph node hyperplasia (Castleman's disease) followed by Kaposi's sarcoma in two homosexual males with the acquired immunodeficiency syndrome (AIDS). Am J Clin Pathol. 1985; 83: 27-33.

8. Frizzera G, Peterson BA, Bayrd ED, Goldman A. A systemic lymphoproliferative disorder with morphologic features of Castleman's disease: clinical findings and clinicopathologic correlations in 15 patients. J Clin Oncol. 1985; 3: 1202-16.

9. Gaba AR, Stein RS, Sweet DL, Variakojis D. Multicentric giant lymph node hyperplasia. Am J Clin Pathol. 1978; 69: 86-90. 